\title{
Research on Dynamic Characteristic of High-Power Magnetostrictive Transducer in high frequency application
}

\author{
Yan Baiping ${ }^{1} \mathrm{a}^{*}$, Zhang Chengming ${ }^{2}$, Li Liyi ${ }^{2}$, Lv Fuzai ${ }^{1}$ \\ ${ }^{1}$ Key Laboratory of Advanced Manufacturing Technology of Zhejiang Province, Hangzhou, China \\ ${ }^{2}$ Department of Electrical Engineering, Harbin Institute of Technology, Harbin, China \\ aEEmail: d_enip@163.com
}

\begin{abstract}
Keywords: Terfenol-D Rod; Magnetostrictive Transducer; Dynamic Characteristic; High Frequency; Abstract. The emphasis of this paper is to study the high frequency dynamic characteristic of highpower transducer based on giant magnetostrictive material. A prototype of high-power magnetostrictive transducer using in $\mathrm{kHz}$ magnitude frequency situation is introduced. The accurate inductance relation in magnetostrictive transducer is calculated, and its dynamic impedance characteristics of the transducer are summarized. Then, the experimental test-bench is set, the impedance-frequency characteristics and high frequency dynamic characteristics are discussed. The results show that magnetostrictive transducer has predominant mechanical strain effect at mechanical resonance situation, its magnetostriction coupling coefficient can up to 0.592 , and has $0.05 \mathrm{~mm}$ output strain at $1200 \mathrm{~Hz}$ frequency. Researches and its results in this paper can helps for the calculation and construction of magnetostrictive transducer using in high frequency application.
\end{abstract}

\section{Introduction}

Electromagnetic Transducer can turn the electric energy into mechanical energy or acoustic energy, has good application in sonar and ultrasonic system. Traditional electromagnetic transducer always has a big volume and mass, and the response time is very slow (always millisecond magnitude), hardly used in the high frequency (likes kHz magnitude frequency) situation [1-2]. Design and construction of electromagnetic transducer based on magnetostrictive materials have received much attention. For magnetostrictive materials, due to the magnetomechanical effect, the change in strain along the axial direction can be produced by the magnetic field, leading to the conversion of the electric energy into mechanical energy [3-4]. Magnetostrictive materials exhibit highly power density and energy conversion efficiency, more suitable to be considered to design smart transducer [5-7].

Today it's still lack of the application of magnetostrictive transducer using in high-power and high frequency $(\mathrm{kHz})$ situation. Researches [8-10] trying to calculate its $\mathrm{kHz}-\mathrm{MHz}$ magnitude frequency application, but the power and structure are very small, hardly to be used in high-power ultrasonic system. Also, the construction and calculation in the high-power transducer are more complex. Its theoretical and experimental calculation in the electrical-mechanical coupling and dynamic characteristics are too simple.

In this paper, a high-power magnetostrictive transducer driving by Terfenol-D rod (a product of GMM) is made which is used in $\mathrm{kHz}$ magnitude frequency situation. The electrical and mechanical characteristics of the transducer are calculated, and the dynamic action and resonant frequency are summarized. Then, magnetostrictive coupling coefficient is analyzed, and hysteresis characteristic is discussed.

\section{Construction of Magnetostrictive Transducer}

The magnetostrictive transducer based on Terfenol-D rod studied has been constructed, and the structure and parameter values are shown in Fig.1. The diameter of Terfenol-D rod is selected as 5mm, and a dimension of $\Phi 5 \times 40 \mathrm{~mm}$ rod is used to design the transducer. The Terfenol-D rod is fixed in the 
middle of transducer by a nylon framework, which is also used to set a solenoids coil outside. The shell is made by high-permeability material (10\# steel).

In magnetostrictive transducer, the solenoids coil, driven by an external current supply, is used to provide magnetic field. Dynamic current in the coil generates a magnetic field along the axial direction, which leads to dynamic strain vibration in the rod direction, turns the electric energy into mechanical energy. The turns of solenoids coil are 688 , and the resistance of coil is $5.06 \Omega$. In transducer, the inductance of coil is $5.3 \mathrm{mH}$.
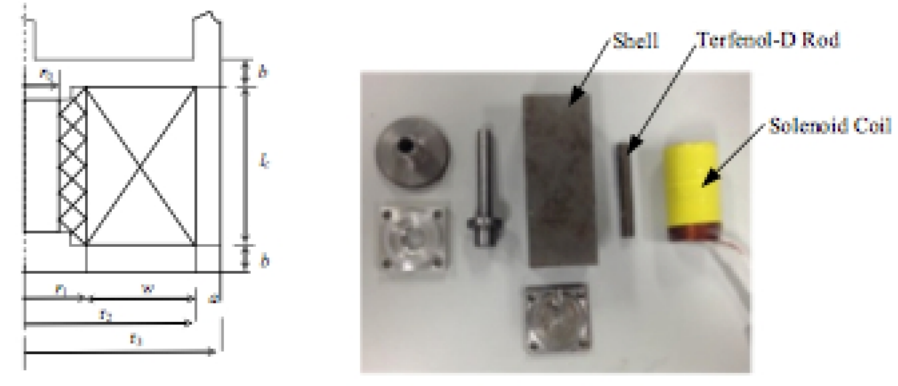

\begin{tabular}{cc}
\hline Parameter & Value \\
\hline Length of Terfenol-D rod $l / \mathrm{mm}$ & 40 \\
Radius of Terfenol-D rod $\mathrm{r} / \mathrm{mm}$ & 2.5 \\
Length of coil $l / \mathrm{mm}$ & 43 \\
Radius of coil w/mm & 6 \\
Turns of coil & 688 \\
Resistance of coil $/ \mathrm{M}$ & 5.06 \\
Inductance of coil $/ \mathrm{mH}$ & 5.3 \\
\hline
\end{tabular}

Fig.1 Geometry structure and basic values of magnetostrictive transducer

\section{Impedance Characteristic of Magnetostrictive Transducer}

In the magnetostrictive transducer, Giant magnetostrictive material (Terfenol-D rod) can turn the magnetic energy into mechanical energy, and external current in the solenoids coil used to provide an axial magnetic field. Its relation can be described as

$$
U=R I+e=R I+\frac{d \psi}{d t}=(R+j \omega L) \cdot I=Z \cdot I
$$

Where $U$ is the input voltage, $I$ is the current in coil. $R$ is the resistance of coil, and $e$ is the back emf in coil induced by the changes of magnetization in transducer. $d t$ is the action time of current. $L$ is the inductance of solenoids coil. $Z$ is the impedance of solenoids coil, and $\omega$ is angular frequency of electrical current. In Equation (1), magnetic flux $\psi$ can also be rewritten as

$$
\psi=N \cdot B S=N S \cdot \mu_{0} \mu_{r} H
$$

In magnetostrictive transducer, as shown in Fig.1, magnetic flux in the transducer can be separated into three parts, Terfenol-D rod region $\psi_{T}$, nylon framework region $\psi_{N}$, and solenoids coil region $\psi_{C}$. The total magnetic flux can be expressed as

$$
\psi=\psi_{T}+\psi_{N}+\psi_{C}=\mu_{0} \mu_{r} N S_{T} H+\mu_{0} N S_{N} H+\psi_{C}
$$

Where $\mu_{0}$ is the vacuum permeability, $\mu_{r}$ is the permeability of Terfenol-D rod. $N$ is the turns of coil. $S_{T}$ and $S_{N}$ are the sectional area of rod and nylon framework. The magnetic flux in solenoids coil region $\psi_{C}$ can be described as

$$
\begin{aligned}
& U=R I+\frac{d\left(\pi \mu_{0} N H \cdot\left[\mu_{r} r_{0}^{2}+\left(R_{1}^{2}-r_{0}^{2}\right)+\int_{R_{1}}^{R_{2}} \int_{R_{1}} \frac{2\left(R_{2}-r\right) r}{\left(R_{2}-R_{1}\right)^{2}} d r d a\right]\right)}{d t} \\
& =\left(R+j \omega \cdot \pi \mu_{0} N^{2} / l \cdot\left[\mu_{r} r_{0}^{2}+\left(R_{1}^{2}-r_{0}^{2}\right)+\int_{R_{1}}^{R_{1}} \int_{R_{1}} \frac{2\left(R_{2}-r\right) r}{\left(R_{2}-R_{1}\right)^{2}} d r d a\right]\right) \cdot I
\end{aligned}
$$

$R_{l}$ and $R_{2}$ are the inter radius and outer radius of solenoids coil, and $l$ is the length of Terfenol-D rod. Compare with equation (1), the inductance of solenoids coil in transducer can be calculated as

$$
L=\pi \mu_{0} N^{2} \cdot\left[\mu_{r} r_{0}^{2}+\left(R_{1}^{2}-r_{0}^{2}\right)+\int_{R_{1}}^{R_{2}} \int_{R_{1}} \frac{2\left(R_{2}-r\right) r}{\left(R_{2}-R_{1}\right)^{2}} d r d a\right] / l=\mu_{0} N^{2} S^{*} / l
$$

As shown in equation (5), the inductance of solenoids coil will be influenced by the permeability $\mu_{r}$ of Terfenol-D rod. Also, equation (5) is the result of inductance in the transducer including Terfenol-D 
rod and outside high-permeability shell. The values will decrease in the structure of no outside highpermeability shell and no inside Terfenol-D rod, as shown in Fig.2. Using equation (5) for calculation, the quasistatic inductance value of solenoids coil in transducer is $5.04 \mathrm{mH}\left(\mu_{r}=10\right.$ is used in its calculation), similarly to its experimental result of $5.15 \mathrm{mH}$.

Fig.2 introduces the inductance-frequency characteristics of solenoids coil in different structure. In this part, three differ structures are used for comparison, structure I: solenoids coil without Terfenol-D rod and shell, structure II: solenoids coil with Terfenol-D rod but no shell, structure III: solenoids coil with Terfenol-D rod and shell. In the low frequency, due to the magnetic flux distribution effect in transducer, magnetic flux $\psi_{T}$ in Terfenol-D rod in structure III is larger than others. So its value of inductance can up $5.15 \mathrm{mH}$, larger than $3.64 \mathrm{mH}$ in structure I. It can be indicated from Fig.2 that there is no obvious value changes happened in the inductance of coil in structure I with frequency increasing. But in structure II and structure III, eddy current loss in Terfenol-D rod will increase with frequency increasing, leads to coefficient $S^{*}$ decrease, and the value of coil inductance also decrease.

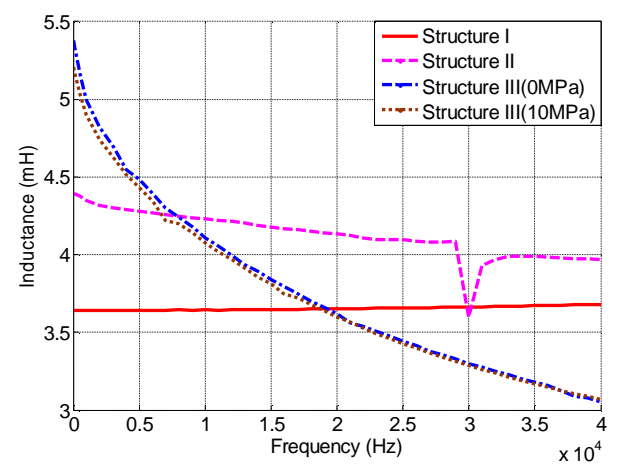

Fig.2 Inductance-frequency characteristics of solenoids coil

Resistance of the coil will increase with the frequency increasing (shown in Fig.3), similarly to the eddy current effect in a hollow copper block. Here must to be indicated that the value of resistance in transducer will changes in differ working frequency, which is always be considered as a invariant parameter in the construction of magnetostrictive transducer. Also, magnetic flux is controlled in the transducer as introduced in Structure III, which will lead to the increase of magnetic leakage in coil section, and its resistance increase coefficient is larger than others.

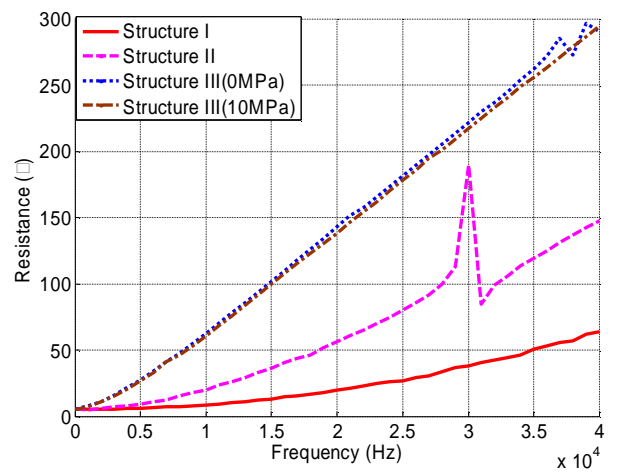

Fig.3 Resistance-frequency characteristics of solenoids coil

As introduced in Fig.2 and Fig.3, an electrical resonance $(29-31 \mathrm{kHz})$ happened in structure II, induced by the decreasing inductance and increasing resistance. The electrical resonance $(37-40 \mathrm{kHz})$ can also saw in structure III with no compress stress situation, but its phenomenon disappeared with the stress loading. And mechanical resonance will have more complex situation, difficult to be predicted in the theoretical analysis. 


\section{High Frequency Dynamic Characteristic of Magnetostrictive Transducer}

In this part, an experimental test bench for the transducer is set, and its dynamic characteristics are analyzed. The results of dynamic effects were introduced in Fig.4-Fig.8, and peak value of dynamic current was selected as 1A. As shown in Fig.4, to obtain continuous 1A peak value current (blue line), the amplitude of voltage (green line) increasing rapidly with the increasing of frequency. At $100 \mathrm{~Hz}$ frequency, it's input voltage just $6.9 \mathrm{~V}$ peak value, but $36.8 \mathrm{~V}$ voltage at $1000 \mathrm{~Hz}$ and $65 \mathrm{~V}$ at $2000 \mathrm{~Hz}$. Besides this effects, the lag angle of current comparing with its input voltage is larger in high frequency than low frequency situation, saw in Fig.5. In the low frequency $(<500 \mathrm{~Hz})$, lag angle enlarges with the increasing of current frequency, and a saturation behavior (value about $71.8^{\circ}$ ) happened when its frequency up to $500 \mathrm{~Hz}$.

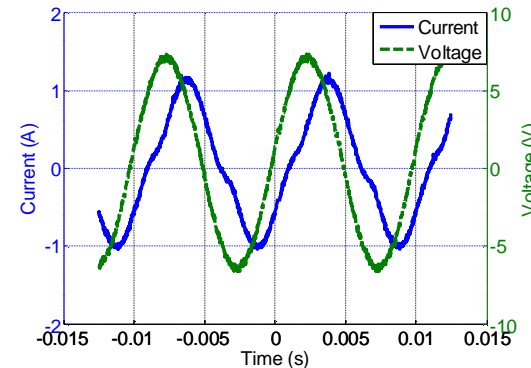

a) Results in $100 \mathrm{~Hz}$ frequency

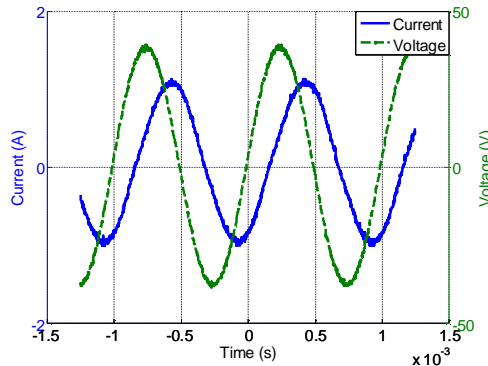

b) Results in $1000 \mathrm{~Hz}$ frequency

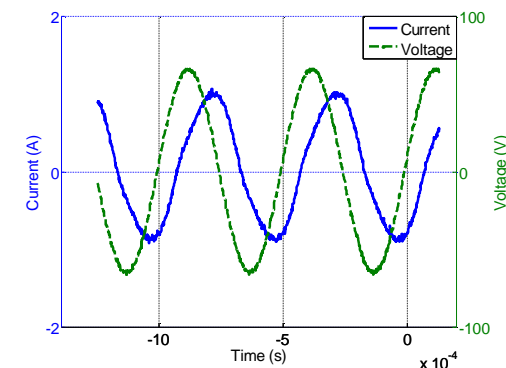

c) Results in $2000 \mathrm{~Hz}$ frequency

Fig.4 Voltage-current curves of magnetostrictive transducer

Also, It can be shown in Fig.5 that a mechanical resonance happened in the frequency of 1100 $1300 \mathrm{~Hz}$, its resonant frequency $f_{\mathrm{r}}$ is $1100 \mathrm{~Hz}$ and anti-resonant frequency $f_{\mathrm{a}}$ is $1300 \mathrm{~Hz}$. Using equation (6) to solve the coupling coefficient of magnetomechanical effect, its value can up to 0.592 (larger than piezoelectric ceramics).

$$
k_{33}=\sqrt{\frac{\pi^{2}}{8}\left(1-\frac{f_{r}^{2}}{f_{a}^{2}}\right)}
$$

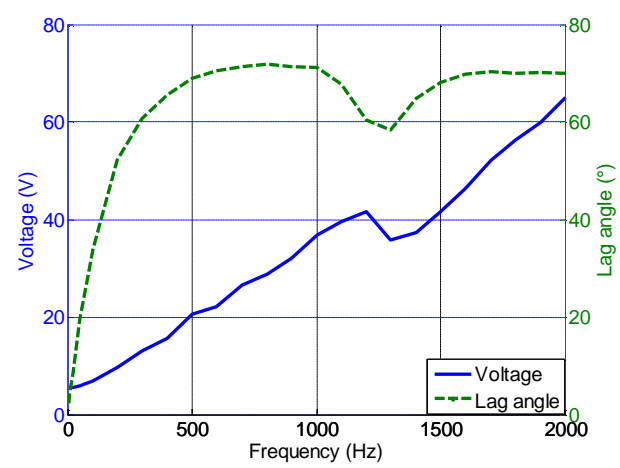

Fig.5 Amplitude of Voltage and lag angle curves in differ frequency

Fig. 6 are the results of resistance and inductance characteristics in transducer. With the increasing of frequency, the eddy current loss in solenoids coil enhances, leads to larger skin effect happened in the section area of coil, which makes resistance increasing. Also, inductance of coil declines with the increasing of frequency, similar to result shown in Fig.2. But the value changes of inductance in Fig.6 are larger than results in Fig.2. Besides eddy current loss effect in Terfenol-D rod introduced in Fig.2, Mechanical vibration (dynamic compress stress) also makes permeability of Terfenol-D rod decline, which leads to larger value changes of inductance about $5-6.5 \mathrm{mH}$ in frequency of $0-2 \mathrm{kHz}$. 


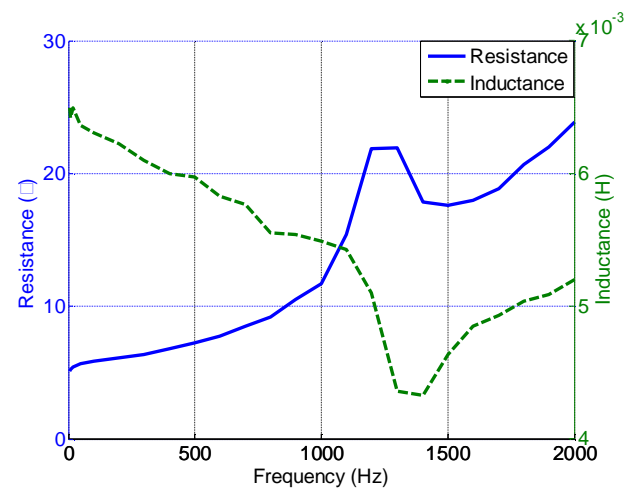

Fig.6 Resistance and Inductance characteristics of transducer

Fig.7-8 introduced the results of mechanical strain characteristics under differ frequency with $1 \mathrm{~A}$ current situation. As shown in Fig.7, in the low frequency, the value of strain remains stable, about $13 \mathrm{~mm}$ output mechanical strain in $1 \mathrm{~A}$ current. When the frequency increasing, close to mechanical resonance frequency of $1100-1300 \mathrm{~Hz}$, transducer has larger output strain, about $0.05 \mathrm{~mm}$ in $1200 \mathrm{~Hz}$. But the output strain is declines fast after mechanical resonance situation, and its value is about $0.005 \mathrm{~mm}$ in $2000 \mathrm{~Hz}$.

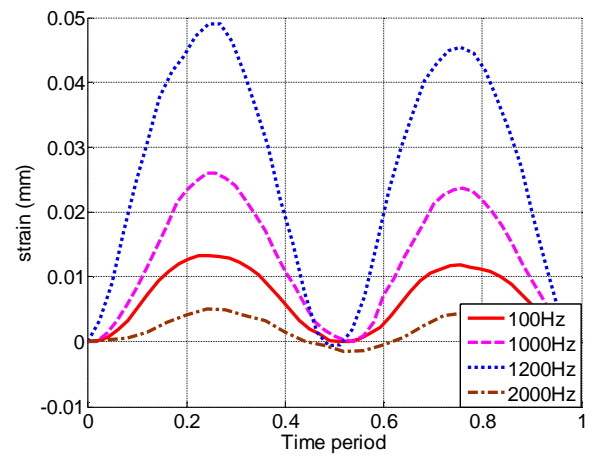

a) dynamic strain of transducer

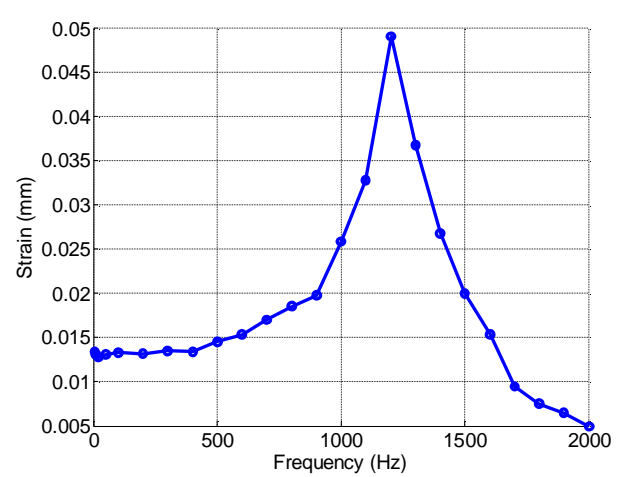

b) amplitude of strain with differ frequency

Fig.7 Dynamic strain characteristics of transducer

Fig.8 introduced the hysteresis effects of strain in transducer. The hysteresis loop of strain is very small at $100 \mathrm{~Hz}$, but larger at $500 \mathrm{~Hz}$. As introduced in Fig.8 that magnetostrictive transducer has larger hysteresis strain characteristics in the high frequency application, which is difficult to predict its dynamic output strain. Also, a mechanical resonance happened in high frequency will helps to turn the electrical energy into mechanical energy (acoustic energy). 


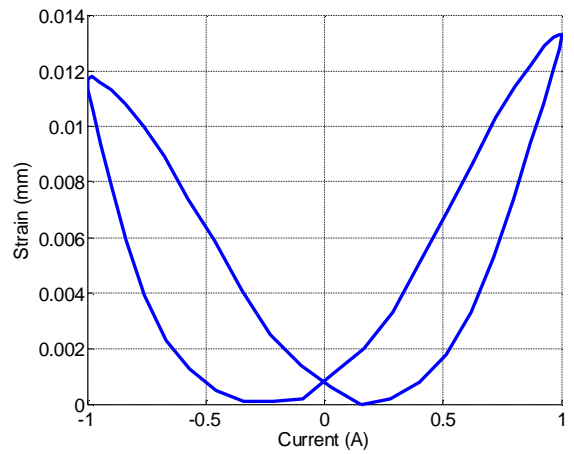

a) results in $100 \mathrm{~Hz}$ frequency

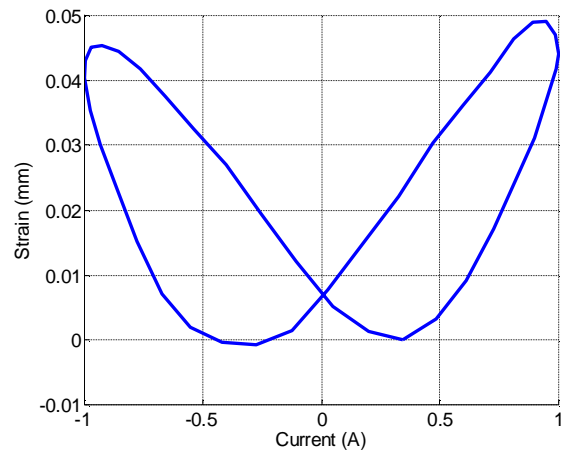

c) results in $1200 \mathrm{~Hz}$ frequency

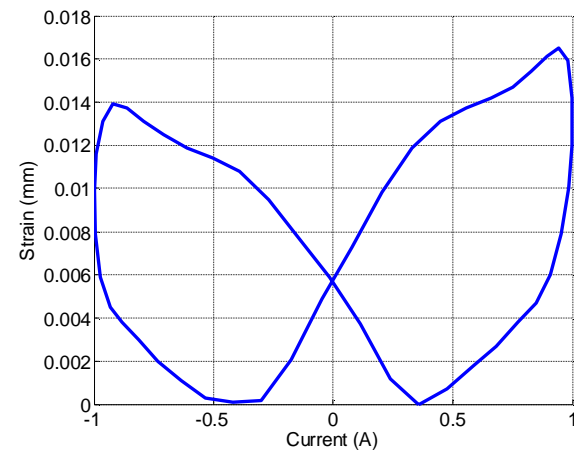

b) results in $500 \mathrm{~Hz}$ frequency

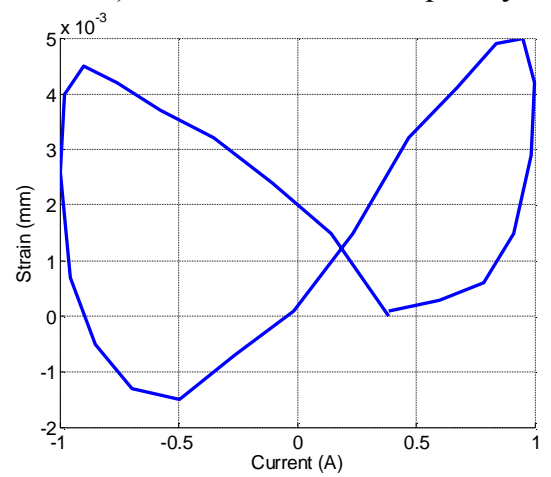

d) results in $2000 \mathrm{~Hz}$ frequency

Fig. 8 Hysteresis characteristics of strain in transducer

Last, the magnetostrictive transducer introduced in this paper can be used in high frequency situation, and a suitable mechanical resonance occurs in the frequency of $1100-1300 \mathrm{~Hz}$. Also, the coupling coefficient of magnetomechanical effect in transducer can up to 0.592 , and it can generate $0.05 \mathrm{~mm}$ output mechanical strain in $1200 \mathrm{~Hz}$ frequency.

\section{Conclusion}

In this paper, a set of transducer based on Terfenol-D rod was designed and its high frequency dynamic characteristics were discussed. The presented transducer can be safely using in the big power and high frequency situation. Its magnetostriction coupling coefficient can up to 0.572 , larger than the piezoelectric transducer. Under the $1100-1300 \mathrm{~Hz}$ Mechanical resonance frequency, the transducer has $0.05 \mathrm{~mm}$ output strain, larger than traditional situation, benefit to its high frequency application design.

\section{References}

[1] H. Varskyi, S. Tankevych, V. Hrechko. Design Features of Electromagnetic Transducer as a Part of Electronic Current Transformer. 2014 IEEE International Conference on Intelligent Energy and Power Systems (IEPS), 2014, pp.60-62.

[2] O. Bottauscio, P. E. Roccato, M. Zucca. Modeling the Dynamic Behavior of Magnetostrictive Actuators. IEEE Trans. Mag, 2010, vol.46, pp.3022-3028.

[3] S. Karunanidhi, M. Singaperumal. Design, analysis and simulation of magnetostrictive actuator and its application to high dynamic servo valve. Sensors and Actuators, 2010, vol.157, pp.185-197.

[4] A. Grunwald, A. G. Olabi. Design of a magnetostrictive (MS) actuator. Sensors and Actuators A, 2008, vol.144, pp.161-175. 
[5] J. Ellison, J. Sirohi, I. Chopra. Design and testing of a bidirectional magnetostrictive-hydraulic hybrid actuator. Smart Structures and Materials, 2004, vol.5390, pp.483-494.

[6] Y. Zhou, S. Priya. Near-flat self-biased magnetoelectric response in geometry gradient composite. Journal of Applied Physics, 2014, vol.115, 104107.

[7] B. Kuanr, R. Camley, Z. Celinski, A. McClure, Y. Idzerda. Single crystal Fe1-xGax thin films for monolithic microwave devices. Journal of Applied Physics, 2014, vol.115, $17 \mathrm{C} 112$.

[8] B. Yoo, S. Na, A. Flatau, D. Pines. Galfenol-Based Directional Magnetostrictive Patch Transducer for Guided Lamb Wave Techniques. Sensors and Smart Structures Technologies for Civil, Mechanical, and Aerospace Systems, 2014, vol.9061, 906146. 